\title{
EQUICONTINUITY, AFFINE MEAN ERGODIC THEOREM AND LINEAR EQUATIONS IN RANDOM NORMED SPACES
}

\author{
V. RADU
}

\begin{abstract}
The aim of this paper is to give a characterization of equicontinuous families of linear operators in random normed spaces which generalizes the normed spaces case.

This characterization is used to generalize some results on iterative solutions of linear equations by using the affine mean ergodic theorem for locally convex spaces (note that every Fréchet space is a random normed space with the $t$-norm $T=\operatorname{Min}$ ).
\end{abstract}

1. Probabilistic metric spaces were introduced by K. Menger [4]. A. N. Šrstnev [11], [12] extended the idea to linear spaces and introduced the notion of random normed space.

In this note is given a characterization of equicontinuous families of linear operators on such spaces. In the second part, this characterization is used in order to generalize a result of Dotson [1] concerning the iterative solutions of linear equations in normed spaces.

Let $S^{1}$ and $S^{2}$ be two random normed spaces [11] and $\mathcal{L}=\mathscr{L}\left(S^{1}, S^{2}\right)$ the family of all linear continuous operators from $S^{1}$ into $S^{2}$, with the $(\varepsilon, \lambda)$ topologies (see also [10]). Let $\Delta^{+}$be the set of all distribution functions $F$ such that $F(0)=0$.

In what follows $H$ will be the element of $\Delta^{+}$defined by

$$
H(x)= \begin{cases}0 & \text { if } x \leqslant 0 \\ 1 & \text { if } x>0 .\end{cases}
$$

If $F$ and $G$ are in $\Delta^{+}$and $F(x) \leqslant G(x)$ for all $x$ in $R$, then we write $F \leqslant G$ or $G \geqslant F$.

We say that a sequence $\left\{F_{n}\right\} \subset \Delta^{+}$is weakly convergent to $H$ if for every $x$ in $(0, \infty), F_{n}(x)$ converges to 1 [9]. In [8] it is proved that a linear operator $f$ : $S^{1} \rightarrow S^{2}$ is continuous if and only if there exists $\nu: \Delta^{+} \rightarrow \Delta^{+}$such that

(i) $\nu$ is weakly continuous at $H$;

(ii) $\nu\left(F_{p}\right) \leqslant F_{f(p)}$.

This result can be extended to families of linear operators in a natural way.

Proposition 1.1. Let $B$ be an equicontinuous family in $\mathcal{L}$. Then there exists $a$ self-mapping $\nu_{B}$ of $\Delta^{+}$such that

(i) $\nu_{B}$ is weakly continuous at $H$;

(ii) $\nu_{B}\left(F_{p}\right) \leqslant F_{f(p)}$ for every $f$ in $B$.

Received by the editors June 5, 1975.

AMS (MOS) subject classifications (1970). Primary 46A15, 47A50; Secondary 47A30, 47A35.

Key words and phrases. Random normed space, equicontinuity, affine mean ergodic theorem, random asymptotically $a$-bounded, random asymptotically $a$-regular. 
Proof. Let $A=\left\{p \in S^{1}, F_{p} \geqslant F\right\}$. It is known [11] that $A$ is a bounded subset of $S^{1}$. Since $B$ is equicontinuous, $\{f(A)\}_{f \in B}$ is uniformly bounded in $S^{2}$. Therefore there exists $G$ in $\Delta^{+}$such that $F_{f(p)} \geqslant G$ for all $f$ in $B$ and $p$ in A.

Let us define

$$
\bar{\nu}_{B}(F)=\inf _{f \in B, p \in A} F_{f(p)} ; \quad \nu_{B}(F)(x)=\sup _{t<x} \bar{\nu}_{B}(F)(t) .
$$

Then it is clear that $\nu_{B}(F)$ is in $\Delta^{+}$and condition (ii) holds. To prove (i) let us assume that there exists a sequence $\left\{F_{n}\right\}$ such that $F_{n}$ converges weakly to $H$ and $\nu_{B}\left(F_{n}\right)$ does not converge to $H$. Then there exist $\varepsilon_{0}$ and $\lambda_{0}$ such that

$$
\forall n \exists m_{n} \geqslant n, \nu_{B}\left(F_{m_{n}}\right)\left(\varepsilon_{0}\right)<1-\lambda_{0} .
$$

Therefore for every $n$ there exist $p_{n}$ in $S^{1}$ and $f_{n}$ in $B$ such that

$$
F_{p_{n}} \geqslant F_{m_{n}} \text { and } F_{f_{n}\left(p_{n}\right)}\left(\varepsilon_{0}\right)<1-\lambda_{0} \text {. }
$$

Since clearly $F_{p_{n}}$ converges weakly to $H$ and $B$ is equicontinuous, $F_{f_{n}\left(p_{n}\right)}$ must converge to $H$, which contradicts (1.2) and the proposition is proved.

REMARK 1. If $B$ is the singleton $\{f\}$ then $\nu_{B}$ is the function $\nu_{f}$ obtained in Theorem 2.1 of [8].

The following theorem is a characterization of equicontinuous families of linear operators.

THEOREM 1.1. Let $B$ be a family of linear operators between two random normed spaces. Then $B$ is equicontinuous if and only if there exists a mapping $\nu$ : $\Delta^{+} \rightarrow \Delta^{+}$such that

(i) $\nu$ is weakly continuous at $H$;

(ii) $\nu\left(F_{p}\right) \leqslant F_{f(p)}$ for every $f$ in $B$.

Proof. For the necessity it suffices to apply the above proposition. To prove the sufficiency let $\left\{p_{n}\right\}$ be convergent to 0 . Then, by the definition, $F_{p_{n}}$ converges weakly to $H$. Since $\nu$ is weakly continuous at $H, \nu\left(F_{p n}\right)$ converges weakly to $H$, and from condition (ii) we obtain that $F_{f\left(p_{n}\right)}$ converges weakly to $H$, uniformly on $f$. Therefore $B$ is equicontinuous.

RemarK 2. From formulas (1.1) it follows that $\nu$ can be supposed nondecreasing. Again from (1.1) and condition (ii) we obtain that $\nu \leqslant \nu_{B} \leqslant \nu_{f}$ for every $f$ in $B$.

Corollary 1. If $S^{1}$ and $S^{2}$ are normed spaces then a family $B$ of linear continuous operators $f: S^{1} \rightarrow S^{2}$ is equicontinuous if and only if there exists $c>0$ such that $\|f\|<c$ for all $f$ in $B$.

Proof. It is known [11] that a normed space can be considered as a random normed space if one sets $F_{p}=H(x-\|p\|)$. By a simple calculation one obtains that $\nu_{f}\left(H_{1}\right)=F_{f}$, where $H_{1}(x)=H(x-1)$. By Remark 2 we have $\nu\left(H_{1}\right) \leqslant F_{f}$ for all $f$ in $B$, which says that $B$ is a bounded subset of $\mathcal{L}$.

Corollary 2. Let $G$ be family of affine mappings $g: S^{1} \rightarrow S^{2}, g=f+q$ where $f$ is linear and $q$ is in $S^{2}$. Then $G$ is equicontinuous if and only if there 
exists a self-mapping $\nu$ of $\Delta^{+}$such that

(a) $\nu$ is weakly continuous at $H$;

(b) $\nu \leqslant \nu_{f}$ for all $f$ in $G$.

Proof. Easy consequence of Remark 2.

THEOREM 1.2. Let $\left\{f_{\alpha}\right\}_{\alpha \in D}$ be a net in $\mathcal{L}$ such that $f_{\alpha}(p)$ converges to $f(p)$ for all $p$ in $S^{1}$. Suppose $S^{1}$ and $S^{2}$ are complete. Then

(i) $f$ is in $\mathrm{L}$;

(ii) there exists $\nu: \Delta^{+} \rightarrow \Delta^{+}$such that

(a) $\nu$ is weakly continuous at $H$;

(b) $\nu \leqslant \nu_{f}$ for every $\alpha$ in $D$.

Proof. It is known that $S^{1}$ and $S^{2}$ are metrizable [12]. Since by hypothesis they are complete, it suffices to apply the uniform boundedness theorem and Proposition 1.1.

2. Let $(S, \mathscr{F}, T)$ be a random normed space with the $t$-norm $T=$ Min. It is easy to see that with the $(\varepsilon, \lambda)$ topology, $S$ is locally convex and metrizable [5]. Let $f: S \rightarrow S$ be a linear and continuous operator.

As in [1] let $a$ be an infinite real matrix, $a=\left[A_{n j}\right]$ such that

$\left(\mathrm{m}_{1}\right) A_{n j} \geqslant 0$ for all $n, j$ and $A_{n j}=0$ if $n<j$;

$\left(\mathrm{m}_{2}\right) \sum_{j=1}^{n} A_{n j}=1$ for each $n$.

Let us define the polynomials $A_{n}$ and $B_{n}$ by

$$
A_{n}(t)=\sum_{j=1}^{n} A_{n j} t^{j-1}, \quad B_{n}(t)=\frac{1-A_{n}(t)}{1-t}
$$

and the linear operators $a_{n}$ and $b_{n}$ by

$$
a_{n}=A_{n}(f), \quad b_{n}=B_{n}(f) .
$$

Definition 2.1. The operator $f$ is said to be random asymptotically $a$ bounded if there exists $\nu: \Delta^{+} \rightarrow \Delta^{+}$such that

(i) $\nu$ is weakly continuous at $H$;

(ii) $\nu \leqslant \nu_{a_{n}}$.

REMARK 3. If $S$ is a normed space and considered as a random normed space (see the proof of Corollary 1) inen $f$ is random asymptotically $a$ bounded iff it is asymptoticall, "unded (see Dotson [1]).

Definition 2.2. The operator $f$ is said to be random asymptotically $a$ regular if the sequence $f a_{n}-a_{n}$ is pointwise convergent to zero.

It is easy to see that if $f$ is random asymptotically $a$-regular then $f^{m}$ is random asymptotically $a$-regular for each $m$ (see [1]). The following proposition generalizes the Lemma 3 from [1]:

Proposition 2.1. Let $a^{i}$ be the identity matrix and $a^{c}$ be the Cesàro matrix. If $f$ is random asymptotically $a^{i}$-bounded then $f$ is random asymptotically $a^{c}$-bounded and random asymptotically $a^{c}$-regular.

Proof. Since $f$ is random asymptotically $a^{i}$-bounded, let $\nu$ be as in Definition 2.1. Then clearly $\nu_{f^{j}} \geqslant \nu$ for all $j$. Since $a_{n}^{c}=n^{-1} \sum_{j=1}^{n} f^{j-1}$, we have 


$$
\begin{aligned}
\nu_{a_{n}^{c}}(F)(x) & =\nu_{n^{-1} \Sigma_{j-1}^{n} f^{j-1}}(F)(x)=\nu_{\sum_{j-1}^{n} j^{j-1}}(F)(n x) \\
& \geqslant \operatorname{Min}_{1<j<n} \nu_{f^{j-1}}(F)(x) \geqslant \nu(F)(x),
\end{aligned}
$$

that is $\nu_{a_{n}^{c}} \geqslant \nu$ for all $n$ which says that $f$ is random asymptotically $a^{c}$ bounded. On the other hand,

$$
\begin{aligned}
F_{f a_{n}^{c} p-a_{n}^{c} p}(x) & =F_{1 / n\left(f_{p-p}^{n}\right)}(x)=F_{f_{p-p}^{n}}(n x) \\
& \geqslant T\left(F_{f_{p}^{n}}(n x / 2), F_{p}(n x / 2)\right) \geqslant T\left(\nu_{f^{n}}\left(F_{p}\right)(n x / 2), F_{p}(n x / 2)\right) \\
& \geqslant T\left(\nu\left(F_{p}\right)(n x / 2), F_{p}(n x / 2)\right)
\end{aligned}
$$

which implies that $f a_{n}^{c} p-a_{n}^{c} p \rightarrow 0$ as $n \rightarrow \infty$, and the proposition is proved.

Let $v$ be an element of $S, f: S \rightarrow S$ a continuous linear operator and consider the equation

$$
\mu-f_{\mu}=v .
$$

We will use the affine mean ergodic theorem of Eberlein-Dotson [1] in order to generalize to random normed spaces some results obtained in [1] concerning the iterative solution of $(2.1)$, in the case of normed spaces. Firstly we will give the affine mean ergodic theorem which is proved in [1] (in a more general case of a locally convex Hausdorff linear topological space). Let $G=\left\{g_{\alpha}\right\}_{\alpha \in I}$ be family of continuous affine self-mappings of $S$.

THEOREM (Dotson [1]). Suppose $\left\{c_{\beta}\right\}$ is a net of continuous affine selfmappings of $S$ such that

(i) $\left\{c_{\beta}\right\}$ is equicontinuous;

(ii) $c_{\beta} r \in \overline{\operatorname{co}}\left(\left\{g_{\alpha} r\right\}_{\alpha \in I}\right)$ for all $\beta$ and $r$.

Suppose $p$ in $S$ such that

(iii) $\lim _{\beta}\left(c_{\beta} g_{\alpha} p-c_{\beta} p\right)=\lim \left(g_{\alpha} c_{\beta} p-c_{\beta} p\right)=0$ for each $\alpha \in I$;

(iv) the net $\left\{c_{\beta} p\right\}$ clusters weakly at $q \in S$.

Then

(v) $g_{\alpha} q=q$ for each $\alpha \in I$;

(vi) $q \in \overline{\operatorname{co}}\left(\left\{g_{\alpha} p\right\}_{\alpha \in I}\right)$;

(vii) $\lim _{\beta} c_{\beta} p=q$.

Let $g=f+v$, where $f$ and $v$ are as in (2.1), let $G=\left\{g^{n}\right\}_{n=0}^{\infty}$ and set $c_{n}=\sum_{j=1}^{n} A_{n j} g^{j-1}$.

THEOREM 2.1. Suppose that $f$ is random asymptotically a-bounded, random asymptotically a-regular and $v$ is in the range of $I-f(I$ is the identity on $S)$. If for some $p$ in $S$ the sequence $\left\{c_{n} p\right\}\left(=\left\{a_{n} p+b_{n} v\right\}\right)$ clusters weakly at the point $u_{p}$, then $\left\{c_{n} p\right\}$ converges to $u_{p}$ which is a solution of the equation (2.1).

PROOF. We will verify the conditions of Dotson's Theorem. In fact we will follow the proof of Theorem 2 in [1].

Since $c_{n}=a_{n}+b_{n} v$ and $f$ is random asymptotically $a$-bounded, (i) is verified. (ii) is clear from the definition of $c_{n}$. To prove (iii) let $q$ in $S$ such that $q-f q=v$. In [1] it is proved that

$$
c_{n} g^{m} p-c_{n} p=g^{m} c_{n} p-c_{n} p=g^{m} a_{n} p-a_{n} p+a_{n} v-a_{n} g^{m} v .
$$

Since $f$ is random asymptotically $a$-regular, condition (iii) is verified. Condi- 
tion (iv) is given by hypothesis and the theorem is proved. By the above Remark 3, Corollary 1 and Corollary 2, this theorem seems to generalize Theorem 2 of [1] and the results obtained from it.

\section{BibLIOGRAPHY}

1. W. G. Dotson, Jr., Mean ergodic theorems and iterative solutions of linear functional equations, J. Math. Anal. Appl. 34 (1971), 141-150. MR 46 \#687.

2. N. Dunford and J. T. Schwartz, Linear operators. I: General theory, Pure and Appl. Math., vol. 7, Interscience, New York, 1958. MR 22 \#8302.

3. V. I. Istrățescu, Introducere în teoria spaţiilor metrice probabiliste și aplicații, Editura Tehnicà, București, 1974.

4. K. Menger, Statistical metrics, Proc. Nat. Acad. Sci. U.S.A. 28 (1942), 535-537. MR 4, 163.

5. D. H. Mustari, On the linearity of isometric mappings of random normed spaces, Kazan, Gos. Univ. Ucen. Zap. 128 (1968), kn. 2, 86-90.

6. O. Onicescu, Nombres et systemes aleatoires, Ed. Acad. Roumaine, Bucharest; Editions Eyrolles, Paris, 1964. MR 30 \#2533.

7. V. Radu, On linear operators in random normed spaces, Bull. Math. Soc. Sci. Math. R. S. Roumanie 17 (1973), 217-220.

8. On a random norm and continuity of linear operators on random normed spaces, $\mathrm{C}$. R. Acad. Sci. Paris 280 (1975), 1303-1305.

9. B. Schweizer, Lectures on P. M-spaces, Lectures Notes, the University of Arizona, 1965 (unpublished).

10. B. Schweizer and A. Sklar, Statistical metric spaces, Pacific J. Math. 10 (1960) 313-334. MR 22 \#5955.

11. A. N. Serstnev, The notion of random normed space, Dokl. Akad. Nauk SSSR 149 (1963), 280-283 = Soviet Math. Dokl. 4 (1963), 388-391. MR 27 \#4268.

12. , Random normed spaces. Questions of completeness, Kazan. Gos. Univ. Ucen. Zap. 122 (1962), kn. 4, 3-20. MR 30 \#239.

Universitatea din Timisoara, Bv. v. Parvan, NR 4, 1900 Timisoara, Romania 